\title{
Immunohistochemical study of vascular injury in acute multiple sclerosis
}

\author{
A J Wakefield, L J More, J Difford, J E McLaughlin
}

\begin{abstract}
Aims-To examine the vascular changes occurring in three archival cases of acute multiple sclerosis, and to provide immunohistochemical evidence of early endothelial cell activation and vascular occlusion in this condition.

Methods-Central nervous system tissues from three cases of acute active multiple sclerosis and six non-inflammatory controls were stained using the following methods: haematoxylin and eosin, Luxol fast blue, cresyl violet, Bielschowsky's silver, and reticulin. Tissues were also immunostained with specific antibodies against collagen type IV, factor XIIIa, class II antigens, glial fibrillary acidic protein, and fibrinogen. Results-Early vascular endothelial cell activation which may progress to vasculitis and vascular occlusion including class II antigen expression and fibrin deposition were identified. The vascular changes were seen prior to cerebral parenchymal reaction and demyelination, and were not seen in control cerebral tissues.

Conclusion-It is proposed that vascular endothelial cell activation may be an early and pivotal event in the evolution of multiple sclerosis, and that demyelination may have an ischaemic basis in this condition. The vascular endothelium may contain an early element in the evolution of multiple sclerosis.
\end{abstract}

(F Clin Pathol 1994;47:129-133)

It is widely accepted that vascular inflammation is a precursor of demyelination in the lesions of multiple sclerosis, and that vasculitis may be a primary event in the evolution of the disease. ${ }^{12}$ Despite this, the vascular injury of multiple sclerosis has received relatively little attention in comparison with the associated demyelinating lesion.

Occlusive vascular changes have been described in multiple sclerosis. Putnam emphasised the prevalence of both acute thrombotic venous occlusion and chronic fibrotic venous obliteration in this disease. ${ }^{3}$ Perivascular and intravascular fibrin deposition are recognised histological features of active lesions in multiple sclerosis, ${ }^{45}$ and unmistakable occlusive changes are seen clinically in retinal vascular lesions during active disease. ${ }^{6}$ Putnam recognised the limitations of evaluating two dimensional histopathological sections when attempting to identify the true prevalence of focal microvascular thrombosis in disease tissues, ${ }^{3}$ which has been confirmed by recent studies of Crohn's disease tissues. ${ }^{78}$ The sequelae of infarction, including haemorrhage and haemosiderin deposition, have also been reported as features of established multiple sclerosis. ${ }^{9}$

Lesions of acute multiple sclerosis are rarely available for evaluation, however, and in chronic lesions thrombus, an impermanent structure, is unusual; therefore an ischaemic pathogenesis for this disease has not been substantiated.

Study of cases of acute, active multiple sclerosis may contribute considerably to our understanding of the early and hence critical phases in the evolution of lesions in this disease.

\section{Methods}

Case one was a 14 year old Afro-Caribbean boy with a history of progressive neurological deficit, which caused his death one month after initial presentation. He had presented with progressive blurring of vision, and increasing confusion. On examination, he had a right afferent pupillary defect and the fundi exhibited bilateral temporal pallor. Both plantar responses were upgoing.

Routine blood tests and serology yielded normal results. Cerebrospinal fluid (CSF) culture and venereal disease tests were negative, and electrophoresis of the cerebrospinal fluid was consistent with local IgG synthesis. Viral studies of both blood and cerebrospinal fluid, and autoantibody studies yielded negative results. A cerebral computed tomography scan showed focal left posterior low attenuation. An electroencephalogram (EEG) demonstrated a well defined left temporoparietal abnormality, which a follow up EEG showed, had gradually spread to the right hemisphere. A clinical diagnosis of acute disseminated encephalomyelitis was made. The patient's condition steadily deteriorated with loss of all brain stem functions and he died one month after onset of clinical disease.

Case 2 was a 29 year old caucasian woman who presented with an acute right hemiparesis and ataxia. A computed tomogram showed a space occupying lesion in the left hemisphere, from which a biopsy specimen was taken at craniotomy. Her subsequent clinical course and electrophoresis results were typical of multiple sclerosis. 
Case 3 was a 19 year old caucasian woman who presented with an acute right hemiparesis and expressive dysphasia. A computed tomogram showed a space occupying lesion in the left anterior parietal region from which a biopsy specimen was taken at craniotomy. Like case 2, she progressed to develop the clinical and cerebrospinal fluid changes consistent with a diagnosis of multiple sclerosis.

\section{ROUTINE HISTOPATHOLOGY}

\section{Case 1}

Sections contained numerous areas of well demarcated demyelination in which surviving axons could be shown. Many lipid laden phagocytes and reactive astrocytes were present. In some sections proliferating astrocytes and microglial cells were present. Perivascular cuffing by lymphocytes, plasma cells, and phagocytes indicated lesions of recent origin. The lesions were widespread, in the cerebrum at the junction of the grey and white matter, and in the corpus callosum, with extensive symmetrical pathology in the brain stem and spinal cord.

\section{Case 2}

Biopsy specimens showed fragments of grey and white matter with well defined areas of demyelination and gliosis. In many places there was a perivascular mononuclear inflammatory cell infiltrate.

\section{Case 3}

Biopsy specimens showed that the brain had been infiltrated with foamy macrophages with reactive gliosis and perivascular lymphocyte cuffing. Occasional plasma cells were seen and axons were preserved.

The changes in all three cases were consistent with a diagnosis of acute multiple sclerosis.

Tissues from these patients were fixed routinely in $10 \%$ formalin and paraffin wax embedded blocks were prepared. Representative sections from these blocks were stained using the following methods: haematoxylin and eosin; Luxol fast bluecresyl violet; Bielschowsky's silver; and Gordon and Sweet's method for reticulin (cases 2 and 3 ). The sections were then examined. Archival material from these cases formed the basis of the present study.

\section{Immunohistochemistry}

From the original paraffin wax embedded material from case 1 , blocks were selected from the medial parietal lobe, including the corpus callosum, the pons midbrain junction, and the upper pons. Biopsy specimens from cases 2 and 3 were taken from the left cerebral hemisphere. Sections were stained immunohistochemically using the following antisera: collagen type IV (vascular basal lamina) (Dako, High Wycombe, Bucks) at a dilution of 1 in 50; factor XIIIa (responsible for cross linking fibrin into stable clots) (Behring, Hounslow, Middx) at a dilution of 1 in 400;
Details of control cases used in study

\begin{tabular}{llll}
\hline Case No & Sex & Age & Central nervous system disease \\
\hline 1 & Female & 11 & Cerebral palsy \\
2 & Male & 42 & Fractured spine \\
3 & Female & 42 & Subarachnoid haemorrhage \\
4 & Female & 38 & Subarachnoid haemorrhage \\
5 & Female & 14 & Down's syndrome \\
6 & Male & 13 & Normal \\
\hline
\end{tabular}

LN3 (class II antigens) (Biotest, Solihull, West Midlands) at a dilution of 1 in 5; and glial fibrillary acid protein (GFAP) (Dako) at a dilution of 1 in 400 .

Sections were digested with trypsin at $37^{\circ} \mathrm{C}$ to produce optimal staining results, and the avidin biotin complex (ABC) method was used to detect the relevant antigen expression.

Fibrinogen (Dako) was detected using the peroxidase-antiperoxidase (PAP) method using antisera at a dilution of 1 in 1000 . Horseradish peroxidase was used as the label, and hydrogen peroxide and diaminobenzidine as the colourisation step. Sections were counterstained with Mayer's haematoxylin.

\section{CONTROLS}

Control cerebral tissues were obtained from six post mortem specimens (table). Blocks from these specimens were stained immunohistochemically, as described.

\section{Results}

\section{ACUTE MULTIPLE SCLEROSIS}

Although the sections from case 1 exhibited a greater spectrum of vascular changes, the appearances described are representative of all three cases.

Vascular changes in the absence of an inflammatory cell infiltrate in the blood vessel wall

The earliest evidence of microvascular injury in acute multiple sclerosis was focal endothelial cell associated fibrin deposition (fig 1A). This was present in many thin walled vessels, including both veins and capillaries. The mural architecture of these was normal, and the fibrin deposition was evident in areas in which both myelin was preserved, and reactive changes in the cerebral parenchyma were absent. Fibrin deposition was not seen in sections from controls. Other vessels seemed to be completely occluded by thrombus (fig 1B).

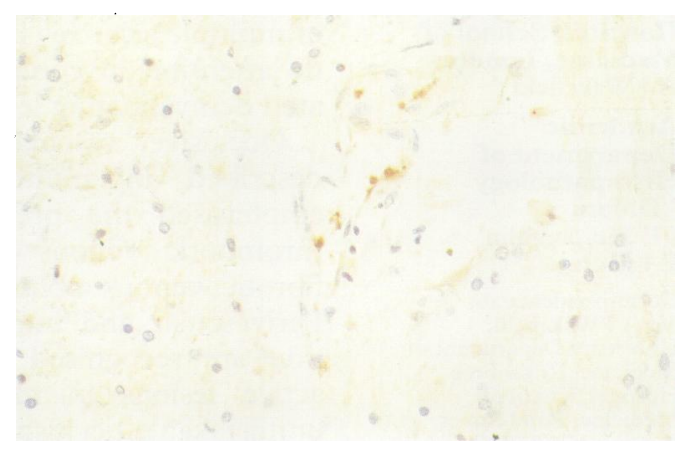

Figure $1 A$ Focal endothelial cell associated fibrin deposition (case 2) (fibrinogen). 


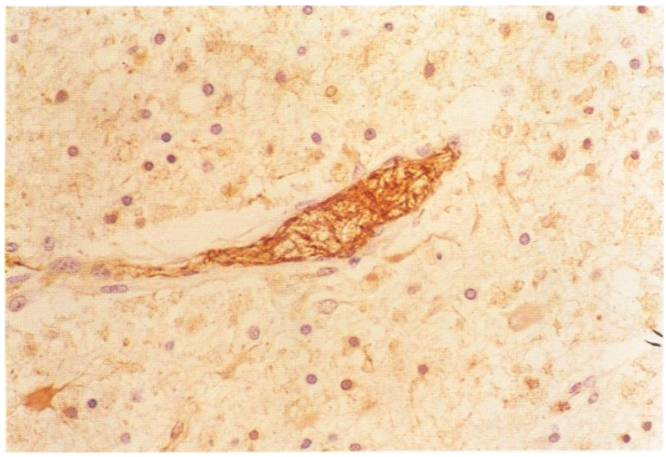

Figure $1 B$ Complete occlusion of vessel by reticulated fibrin thrombus. Although the background is greater than in fig $1 A$, the characteristic reticulated pattern is indicative of fibrin deposition (case 1) (fibrinogen).

Vessels showing these and more advanced vascular changes were often grouped together, suggesting that the microcirculation of discrete areas of the brain was compromised. In case 1 lesional areas of vascular occlusion were noted to have a symmetrical

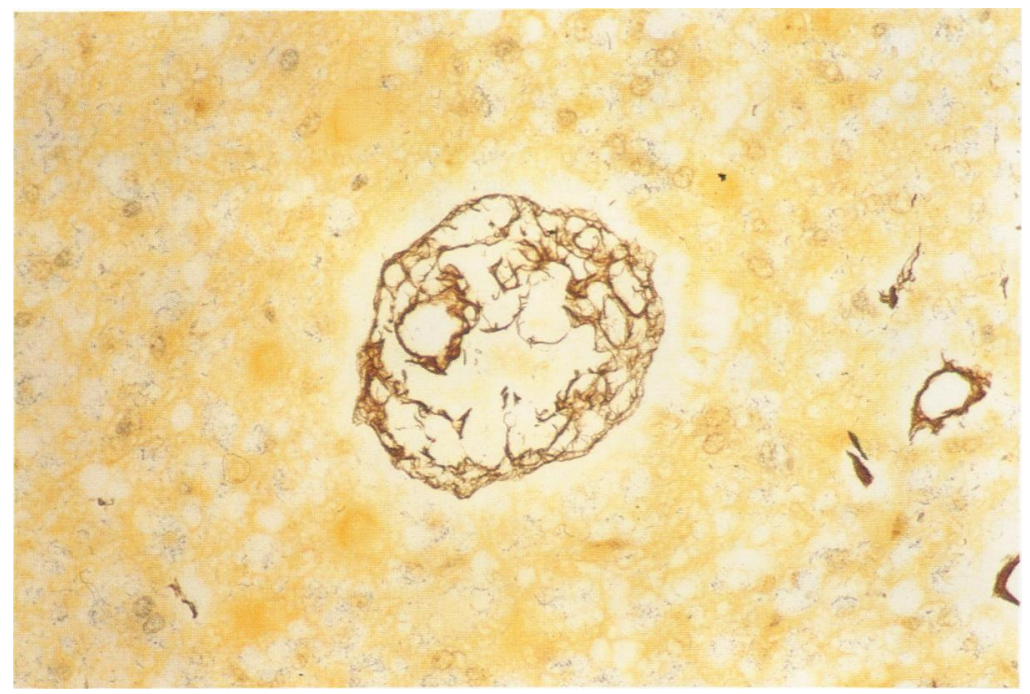

Figure $2 A$ Progressive disruption of the vessel wall in advanced vascular injury (case 3) (reticulin).

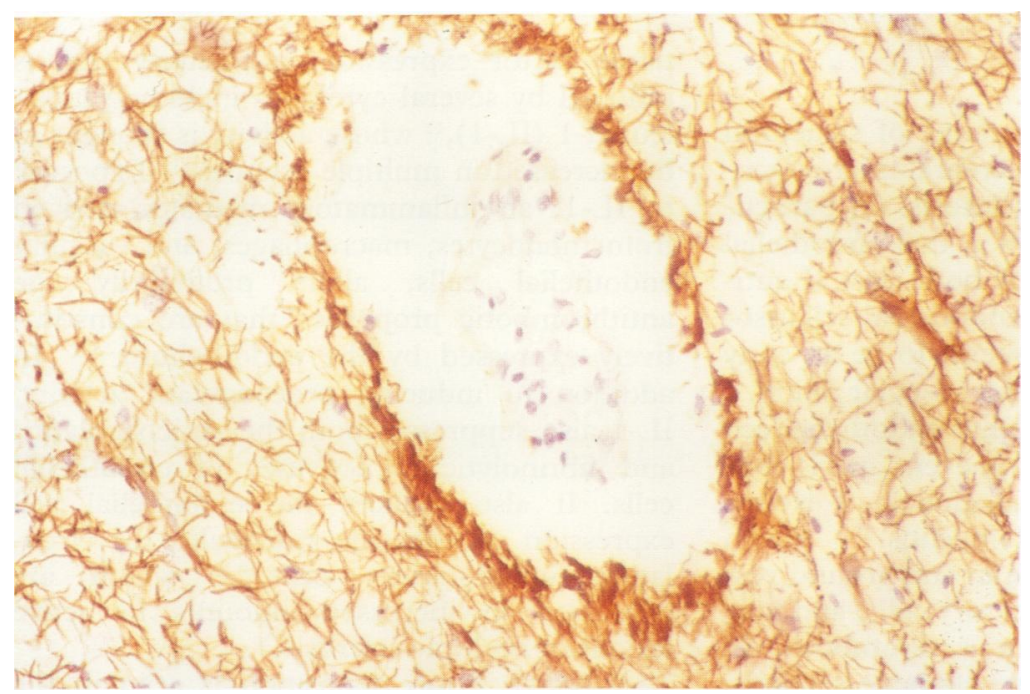

Figure $2 B$ Relation of the glial limitans to the vessel wall. Early infiltration of the vessel wall by chronic inflammatory cells: the outer vessel wall is separated from the glial limitans by the perivascular space (case 2) (glial fibrillary acidic protein).

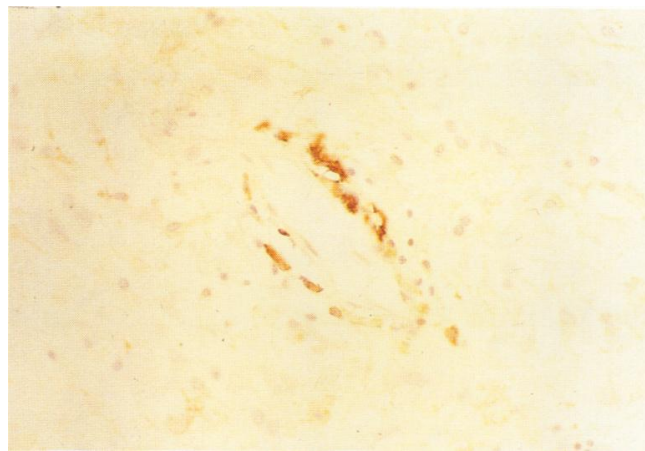

Figure 1C Class II antigen expression by vascular cells including endothelium in an area of histologically "normal" white matter that was not seen in control cases (case 1) (LN3).

distribution in one transverse section of the brainstem.

In areas of vascular injury, which were separated sharply from unaffected areas, small venules, capillaries, and occasional cells within the vessel walls exhibited positive immunostaining for class II antigen expression (fig 1C), a feature that is restricted to activated endothelium. ${ }^{10}$ These findings were also present in areas where vessels exhibited normal mural architecture, and there was little evidence of cerebral parenchymal reaction. The immunohistochemical changes described above were not present in sections of control tissues.

Advanced vascular changes associated with an intramural inflammatory infiltrate

In acute multiple sclerosis progressive vascular injury is associated with reduplication of the vascular basal lamina around an infiltrate of chronic inflammatory cells, and disruption of the blood vessel wall, shown by staining for either collagen type IV or reticulin (fig $2 \mathrm{~A}$ ). The inflammatory infiltrate seemed to be restricted, at least initially, to the blood vessel wall in these lesions. In more advanced lesions extension of the inflammatory infiltrate to the perivascular space was apparent. These findings were reinforced by the results of immunostaining for glial fibrillary acid protein, which showed that the inflammatory infiltrate in the vessel wall was within, but not enclosed by, the glial limitans (fig 2B).

Haematoxylin and eosin stained sections showed that these appearances were often accompanied by extravasation of red cells into the wall of the vessel (fig 3A) and in some areas by frank intracerebral haemorrhage (fig 3B).

Both intramural and perivascular fibrin deposition were evident in advanced vascular injury (figs $4 \mathrm{~A}$ and $\mathrm{B}$ ), and the walls of these vessels appeared greatly widened by inflammatory infiltrate and oedema. These more advanced vascular changes were accompanied by the presence of reactive astroglial cells around the damaged vessels.

In the more advanced lesions identified in sections from case 1 groups of vessels were apparently obliterated by a material that exhibited intense staining for factor XIIIa 


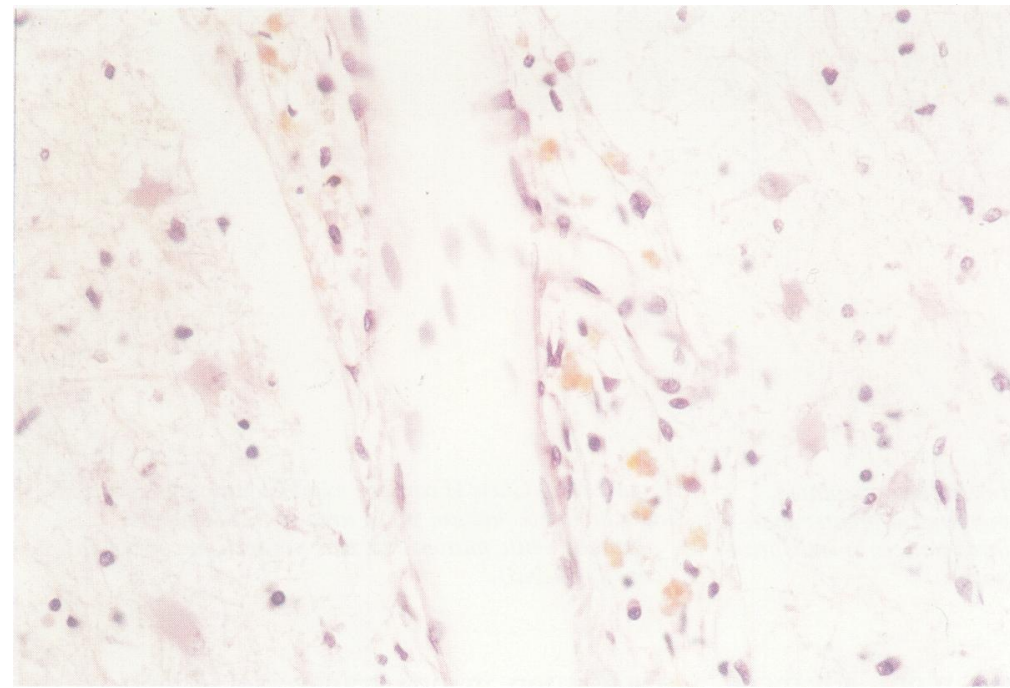

Figure $3 A$ Vasculitis associated with intramural haemosiderin deposition (case 2) (haematoxylin and eosin).

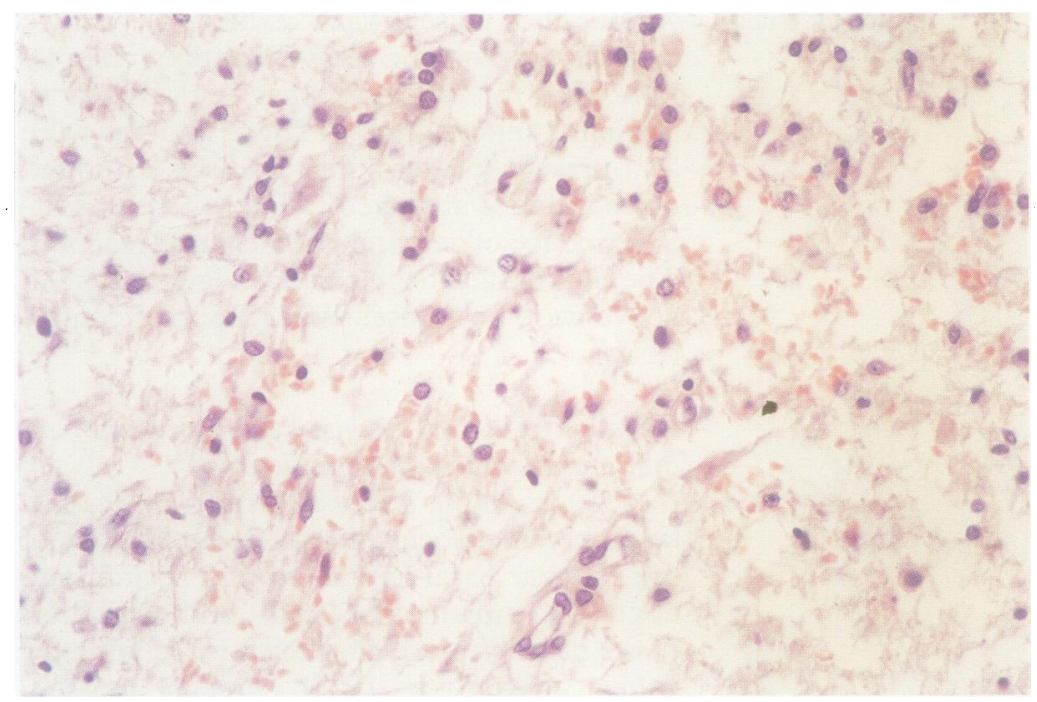

Figure $3 B$ Clinically evident intracerebral haemorrhage at the level of the pontomidbrain junction (case 1) (haematoxylin and eosin).

(fig 4C); the vascular origin of these lesions was confirmed by collagen type IV immunostaining on serial section.

\section{Discussion}

This study shows that a range of structural abnormalities affects the cerebral microvasculature in acute multiple sclerosis. Of particular interest is the observation that endothelial cell activation, manifest by both class II antigen expression and focal endothelial cell associated fibrin deposition, seems to be an early event in the evolution of this vascular injury.

These features suggest that endothelial cell activation may be a primary event and is consistent with certain animal models of experimental allergic encephalomyelitis. ${ }^{1011}$ Fibrin persists subsequently both in and around vessels infiltrated by inflammatory cells, and may progress to occlusive venous thrombosis. In haematoxylin and eosin stained sections, haemorrhage was frequently seen in relation to these damaged vessels.

Fibrin deposition in cell mediated immune responses may be initiated by endothelial cell

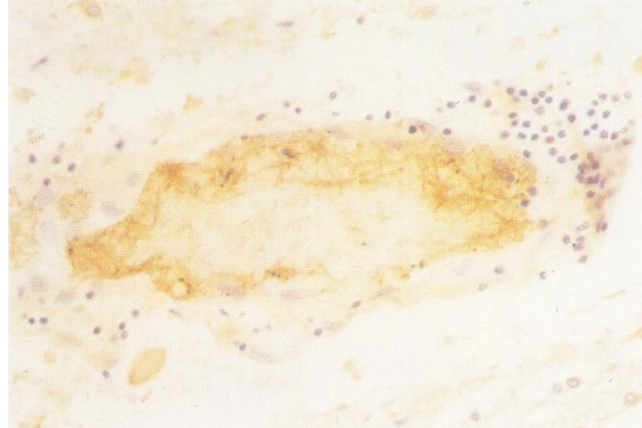

Figure 4A Fibrinous occlusion of an inflamed vessel (case 1) (fibrinogen).

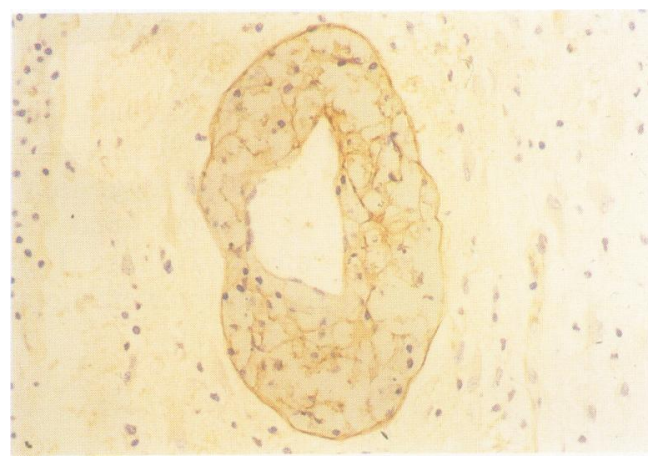

Figure 4B Cross linked intramural fibrin deposition in blood vessel exhibiting advanced inflammatory changes (case 1) (factor XIIIa).

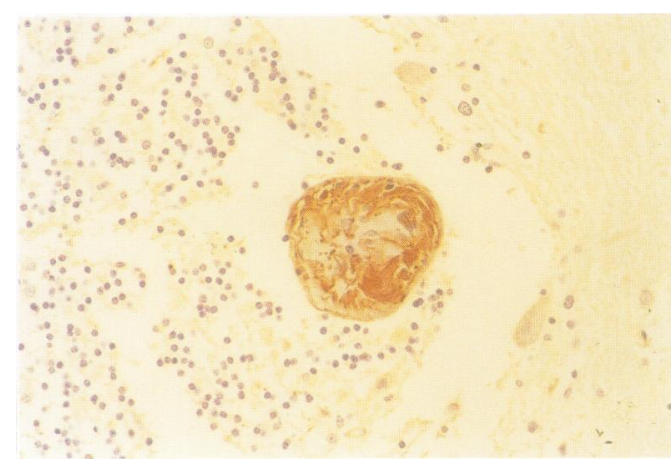

Figure 4C Obliterative vascular injury in lesion of the cerebellum. Collagen type IV immunostaining in serial section confirmed the vascular origin of this structure (case 1) (factor XIIIa).

tissue factor expression, a pathway that is induced by several cytokines including interleukin-1 (IL-1), ${ }^{12}$ whose activity is thought to be increased in multiple sclerosis. ${ }^{13}$ Exposure to $\mathrm{IL}-1$, an inflammatory mediator derived from monocytes, macrophages, and vascular endothelial cells, alters profoundly the antithrombotic properties that are constitutively expressed by the endothelium. ${ }^{14-17}$ In addition to inducing procoagulant activity, IL-1 also suppresses both the anticoagulant ${ }^{14}$ and fibrinolytic ${ }^{16,17}$ activities of endothelial cells. It also induces the endothelial cell expression of adhesion molecules for circulating inflammatory cells, ${ }^{18} 19$ which are expressed in increased amounts in active lesions of multiple sclerosis. ${ }^{20}$

Although fibrin deposition is seen in the vascular lesions of "active" multiple sclerosis, it is not typical of chronic lesions. ${ }^{3-5}$ These differences suggest that the formation and dissolution of fibrin may be part of the nat- 
ural history of the early stages of the disease. In certain models of experimental allergic encephalomyelitis fibrin formation within lesions seems to be a prerequisite for the development of clinical disease. ${ }^{10}$ Interestingly, disease activity began only with the appearance of fibrin in this model; neither paralysis nor fibrin deposition were temporally related to perivascular cellular infiltration, a feature which persisted beyond the period of clinical recovery. ${ }^{10}$

Could focal cerebral ischaemia account for the pathological and clinical features of multiple sclerosis? Demyelination with a relative preservation of axis cylinders was observed in experimental models of cerebral microvascular occlusion and cerebral hypoxic injury. ${ }^{21}$ Leucoencephalopathy characterised by focal demyelination with preservation of axon cylinders is a recognised feature of certain forms of cerebral hypoxia in man. ${ }^{22}$ Preferential demyelination in certain forms of ischaemic and hypoxic injuries is a feature of these cases, and may account for the pattern of histological changes seen in multiple sclerosis.

In an archival case of cerebral malaria, a recognised microangiopathic condition, we showed that in areas of perivascular demyelination axons are preserved (unpublished data). In multiple sclerosis the localisation of vascular injury to areas of white matter occurs at a distance from the metabolically active nerve cell body, but involves the oxygen dependent cell bodies of oligodendroglia. Furthermore, it may be that the morphology of lesions associated with multiple sclerosis, a chronic venulitis, would be different from those of an acute arterial lesion, which is by far the most common cause of vascular injury to the central nervous system.

Provocation of symptoms in response to a hot bath, exercise, food and alcohol are features of multiple sclerosis. ${ }^{23}$ Delayed impulse propagation and synaptic transmission may be features of both ischaemia and multiple sclerosis $^{24}$ 25; experimental neural ischaemia may produce both conduction block and focal myelin damage. ${ }^{25}$ Both deterioration in neural activity following exposure to the provoking stimulus, and subsequent clinical improvement following its removal, are rapid. All of these provocative stimuli share a common denominator-namely, vasodilatation of a vascular bed that is distinct from that of the central nervous system. The fact that foci of blood brain barrier injury in multiple sclerosis, identified by proton magnetic resonance imaging, are associated with a lactate peak, also suggests an ischaemic process. ${ }^{26}$ Despite autoregulation, which might be impaired in areas of vascular injury, shunting of even small amounts of blood away from critically perfused lesions is likely to produce an acute symptomatic deterioration. Restoration of blood flow following removal of the stimulus would produce a rapid clinical improvement.

This immunohistochemical study has described some of the microvascular changes occurring in three cases of acute multiple sclerosis. We suggest that focal endothelial cell activation which progresses to occlusive vascular inflammation is a precursor of both cellular infiltration of vessels and demyelination. Advanced vascular injury is associated with infiltration of the vessel wall by inflammatory cells and reactive changes in the neuropil. We propose that activation of the cerebral endothelium is a primary event in multiple sclerosis; that induction of procoagulant activity in endothelial cells is a feature of acute multiple sclerosis; and that demyelination may have an ischaemic basis in this disease.

We are indebted to Miss Doris Elliott for her help in the preparation of this manuscript. Mr A J Wakefield is supported by the Wellcome Trust.

1 Compston DAS. The dissemination of multiple sclerosis. f $R$ Coll Physicians Lond 1990;24:207-18.

2 Lightman S, McDonald WI, Bird AC, et al. Retinal venous sheathing in optic neuritis. Brain 1987;110: 405-14.

3 Putnam TJ. Evidences of vascular occlusion in multiple sclerosis and encephalomyelitis. Arch Neurol Neuropsycho 1935;32:1298-321

4 Adams C. A colour atlas of multiple sclerosis and other myelin disorders. London: Woolfe, 1989:101-3.

5 Putnam TJ. Lesions of encephalomyelitis and multiple sclerosis: venous thrombosis as the primary alteration fAMA 1937;108:1477-84

6 Graham EM, Stanford MR, Sanders MD, Kasp E, Dumonde DC. A point prevalence study of 150 patients with idiopathic retinal vasculitis: 1 . Diagnostic value of ophthalmological features. $\mathrm{Br} \mathcal{F}$ Ophthalmol 1989;73
714-21.

7 Wakefield AJ, Sawyerr AM, Dhillon AP, et al. Pathogenesis of Crohn's disease: multifocal gastroinPathogenesis of Crohn's disease: multifoct
testinal infarction. Lancet 1989;i:1057-62.

8 Wakefield AJ, Dhillon AP, Sawyerr AM, et al. Granulomatous vasculitis in Crohn's disease. Gastroenterology 1991;100:1279-87.

9 Adams CWM. Perivascular iron deposition and other vascular damage in multiple sclerosis. $\mathcal{F}$ Neurol Neurosurg Psychiatry 1988;51:260-5.

10 Paterson PY. Experimental allergic encephalomyelitis: role of fibrin deposition in immuropathogenesis of inflammation in rats. Fed Proc 1976;35:2428-33.

11 Sobel RA, Blanchette BW, Bhan AK, Colvin RB. The immunopathology of experimental allergic encephalomyelitis. F Immunol 1984;132:2402-7.

12 Bevilaqua MP, Pober JS, Majeau GR, Cotran RS, Gimbrone MA. Interleukin-1 (IL-1) induces biosynthesis and cell surface expression of procoagulant activity in sis and cell surface expression of procoagulant activity in 160:618-23.

13 Selmaj K, Nowak Z, Tchorzewski H. Multiple sclerosis: effect of myelin basic protein on interleukin-1, interleukin-2 production, and interleukin-2 receptor expression in vitro. Clin Exp Immunol 1988;72:428-33.

14 Narworth PP, Handley DA, Esmon CT, Stern DM Interleukin-1 induces endothelial cell procoagulant activity while suppressing cell surface anticoagulant activity. Proc Natl Acad Sci USA 1986;83:3460-4.

15 Emeis J, Kooistra T. Interleukin-1 and lipopolysaccharide induce an inhibitor of tissue-type plasminogen activator in vivo and in cultured endothelial cells. $f$ Exp Med 1986;163:1260-6.

16 Nachman RL, Hajiar KA, Silverstein RL, Dinarello CA Interleukin-1 induced endothelial cell synthesis of plasminogen activator inhibitor. $f$ Exp Med 1986;163: minogen

17 Bevilaqua MP, Scheelf RR, Gimbrone MA, Loskutoff DJ. Regulation of the fibrinolytic system of cultured human vegulation of the fibrinolytic system of cultured human vascular endoth

18 Pober JS. Cytokine mediated activation of the vascular endothelium. Am $\mathcal{F}$ Pathol 1988;133:426-33.

19 Mantovani A, Dejana E. Cytokines as communication sig nals between leucocytes and endothelial cells. Immunol Today 1989;10:370-5

20 Traugott N, Frohman E, Scheinberg LC. Role of adhesion molecules in the immunopathogenesis of multiple sclerosis lesions. Neurology 1989;39(suppl 1):171-6.

21 Putnam TJ, McKenna JB, Morrison LR. Studies in multiple sclerosis: the histogenesis of experimental sclerotic plaques and their relation to multiple sclerosis. fAMA 1931;97:1591-6.

22 Ginsberg MD, Hadley-White ET, Richardson EP Hypoxic ischaemic leucoencephalopathy in man. Arch Neurol 1976:33:5-14.

23 Halliday AM, McDonald WI. Pathophysiology of demyelinating disease. Multiple sclerosis. Br Med Bull 977;33:21-7.

24 Gibson G, Shimada M, Blass JP. Alterations in acetylcholine synthesis and cyclic nucleotides in mild cerebral choline synthesis and cyclic nucleotides

25 Wright EB. The effects of asphyxiation and narcosis on peripheral nerve polarization and conduction. $A m \tilde{J}$ Physiol 1947;148:174-84.

26 Miller DH, Austin SJ, Connelly A. Proton magnetic resonance spectroscopy of an acute and chronic lesion in multiple sclerosis. Lancet 1991;i:380-6. 